\title{
ATTENTION MAINTENANCE IN NOVICE DRIVERS: ASSESSMENT AND TRAINING
}

Anuj Pradhan, Kathleen M. Masserang, Gautam Divekar, Ian Reagan, F. Dennis Thomas, Richard Blomberg, Alexander Pollatsek \& Donald Fisher University of Massachusetts at Amherst Amherst, MA, USA E-mail: apradhan@ecs.umass.edu

\begin{abstract}
Summary: All programs assessing attention maintenance inside the vehicle have required eye trackers and either a driving simulator or a specially equipped field vehicle. Ideally, one would like a way to assess attention maintenance that could be implemented on a desktop PC. Additionally, one would like to have a program that could be used to train novice drivers to maintain their attention more safely on the forward roadway. An experiment was run (a) to determine whether a program FOCAL (Focused Concentration and Attention Learning) using a desktop PC could differentiate between the attention maintenance skills of novice and experienced drivers and (b) to determine whether a program that improved the hazard anticipation skills of novice drivers might also improve their attention maintenance skills. FOCAL was able to differentiate between the attention maintenance skills of novice and experienced drivers. However, hazard anticipation training did not improve the attention maintenance skills of the novice drivers.
\end{abstract}

\section{INTRODUCTION}

Distraction has long been recognized as a major contributor to automobile crashes among all drivers (Wang, Knipling, and Goodman, 1996). The magnitude of the problem is likely to increase because of the growing popularity of in-vehicle tasks that require the driver to glance away from the forward roadway frequently and for long periods of time - most notably music retrieval operations (Chisholm, Caird and Lockhart, 2008; Salvucci, Markley, Zuber, and Brumby, 2007) and text messaging with cell phones (Lerner and Boyd, 2004; Strayer, Drews and Crouch, 2003).

A recent naturalistic study of drivers in the field suggests just how risky even brief glances away from the forward roadway can be (Klauer, Dingus, Neale, Sudweeks \& Ramsey, 2006). In this study, the total time that drivers' eyes were off the forward roadway was computed for critical epochs of six seconds: from five seconds preceding an event that defined a crash or near crash until one second after the defining event. The total time the eyes were off the forward roadway was also computed for baseline six second epochs where there was no crash or near crash. Finally, the odds ratio was computed: the numerator was the odds defined by the ratio of the number of six second crash/near crash epochs with glances away from the forward roadway for more than two seconds to the number of six second crash/near crash epochs with glances away from the forward roadway for less than two seconds; the denominator was the same odds for baseline epochs. The odds ratio in this case was 2.19 (significantly greater than 1.0) 
Research on a driving simulator and in the field suggests that teens are especially likely to glance away from the forward roadway for periods of time longer than two seconds. In a study undertaken on a simulator (Chan, Pradhan, Knodler, Pollatsek \& Fisher, 2008), newly-licensed (16-18) and experienced (21 years old and older) drivers were asked to navigate through a virtual world while undertaking at various points in time one of five secondary tasks inside the vehicle (e.g., looking for a $\mathrm{CD}$ on the front seat). Perhaps the simplest measure of sustained inattention is the mean length of the maximum glance (the maximum period of time a driver spent with his or her eyes continually off the road during an in-vehicle task) averaged over the five in-vehicle scenarios for each driver. There were large differences between the older and younger drivers on this measure: 1.63 seconds for the older drivers and 2.76 seconds for the younger drivers. However, because an average measure may overweight one or two extremely long glances, the percentage of scenarios for each participant in which the maximum episode was over various thresholds was also computed. There were large differences between the two groups on these measures as well. The percent of scenarios in which there was glance of over 2 seconds was $20 \%$ for the older drivers and $56.7 \%$ for the younger drivers. There were also large differences for cutoffs at 2.5 seconds $(10.0 \%$ vs. $45.0 \%)$ and 3 seconds $(6.7 \%$ vs. $33.3 \%)$. Finally, although the total time that the younger drivers spent with their eyes off the road for the in-vehicle tasks (7.36 s) was somewhat longer than for older drivers $(5.80 \mathrm{~s})$, this difference was not statistically significant. Thus, the major difference between the groups was not so much the total time that their eyes were off the road, but how they distributed it.

In a field study in Finland (Wikman, Nieminen \& Summala, 1998), much the same results were reported. Twenty-three experienced and 24 inexperienced drivers drove a $126 \mathrm{~km}$ course through city streets and rural roads. Although the average duration of a glance did not differ significantly as a function of experience (consistent with Lee, Olsen, \& Simons-Morton, 2006), the distribution of the glance durations did differ quite a bit. Only $13 \%$ of the experienced drivers had in-car glance durations of at least $2.5 \mathrm{~s}$ whereas $46 \%$ of inexperienced drivers had in-car glance durations at least this long. Similarly, $0 \%$ of the experienced drivers had in-car glance durations of at least $3 \mathrm{~s}$, whereas $29 \%$ of the inexperienced drivers did.

Given the above results, it appears that current driver education programs are not giving novice drivers an understanding of just how dangerous long glances are away from the forward roadway. In order to deal with the problem, we need a practical method to assess novice drivers' attention maintenance skills that does not rely on expensive equipment such as eye tracking equipment. This method must achieve a real measure of face, construct and predictive validity in order to be considered useful. There is also a clear need for a program to improve the attention maintenance skills of novice drivers. Ideally, one could use a training program that was already available. The development a new attention maintenance assessment method and the use of this method to evaluate an existing training program are described below.

\section{METHOD}

There are several possible ways one might gather information on the duration of a driver's glances away from the forward roadway without actually tracking the driver's eye movements. We decided to ask the driver to toggle between the top half of a screen that contained a view of the roadway and instrument panel (Figure 1, left) and the bottom half that contained a map 
(Figure 1, right). We recorded the time that the participant looked at the map whenever it replaced the view of the forward roadway. Although one could construct a new program specifically targeted at the training of attention maintenance, we decided to test whether our existing hazard anticipation program (Risk Awareness and Perception Training program or RAPT-3) that greatly increased novice drivers' ability to anticipate hazards both on a driving simulator (Pollatsek, Narayanaan, Pradhan, \& Fisher, 2006) and on the open road (Pradhan, Pollatsek, Knodler \& Fisher, 2009) would also decrease these drivers' willingness to take their eyes away from the forward roadway.

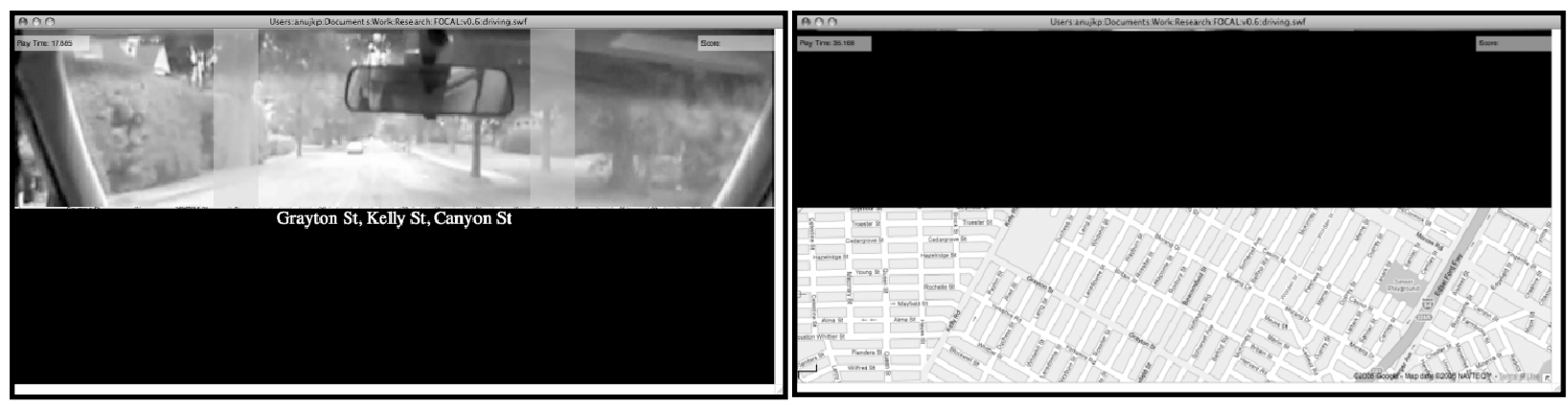

Figure 1. Forward View (left panel). Map View (right panel)

Participants. The participants were 23 younger drivers between the ages of 16 and 18 who were taking a driver education class and 11 experienced drivers between the ages of 35 and 55 .

Attention Maintenance Assessment Program (FOCAL). Four videos, each approximately one minute in length, were filmed throughout downtown Amherst from the perspective of the driver. There were street signs, traffic signs, pedestrians and traffic throughout. The videos were formatted and embedded into a web player. The program allowed for a split screen, where the top half of the screen displayed the video and the bottom half displayed a map. While viewing half of the screen, the other was blacked out (Figure 1). The subject had to press the spacebar to see the map and then hit the enter key to toggle back up to the playing video. Each trial began with the forward (video) view. While viewing the video, the subjects' task was to press the 'enter' key to indicate that a traffic sign, pedestrian or opposing vehicle was passing in front of the vertical light gray bars that were superimposed on the forward view (Figure 1, left panel). While viewing the map, the subjects' task was to search for three street names presented at the beginning of the trial. At the end of the trial, the participant reported which of the street names were present. The evaluation tool will be referred to as FOCAL (FOcused Concentration and Attention Learning).

Hazard Anticipation Training Program (RAPT). The hazard anticipation training program (RAPT-3) has been described at length elsewhere (e.g., Pradhan et al., 2009). Briefly, participants were shown a sequence of still photographs each displayed for 3 seconds. Each sequence was filmed in downtown Amherst in areas which contained situations in which a hidden threat might emerge from behind some obscuring object (e.g., a pedestrian might emerge from behind a truck stopped in the parking lane right before a marked midblock crosswalk) or a stationary vehicle might become a potential threat (e.g., a vehicle in a left turn lane might suddenly pull into the adjacent lane in front of the driver). The participants must click on all 
areas of each photograph where they would look if they were actually driving through the scene depicted. If a driver did not click in the area that was of most concern, a plan view was presented describing the potential threat and the participant repeated the sequence of stills until he or she clicked in the correct area or five repetitions had elapsed.

Design. Younger drivers were assigned randomly either to the RAPT (training with the risk awareness and perception training program, RAPT-3; a total of 11) or no-RAPT group (no training; a total of 12). Older drivers received no training. RAPT training always preceded the test of attention maintenance. The videos were presented in the same order to all participants.

Procedure. Before beginning experimental trials, participants were given five practice trials. Subjects were instructed to perform the tasks as though they were the driver in the video and to weight the tasks equally, but consistent with safe driving. In the practice trials, participants were trained on each task (toggling between map view and video view, map search task, video task) individually before performing them simultaneously for the last two practice trials. For each of the four videos, the three street names appeared just below the top screen in the video view (Figure 1, left). (This was to minimize reliance on memory.) At the end of each video, the participant had to indicate which of the three street names he or she had identified.

\section{RESULTS}

The measure we focused on was the length of a glance away from looking at the forward roadway: the amount of time between when the participant pressed the spacebar to view the map and when they pressed the enter key to view the roadways again. The simplest single global measure of extended inattention to the forward roadway is the average of these glance durations. The averages were substantially higher for the younger no-RAPT group and the younger RAPT group (4.12s and 4.06s, respectively) than for the older experienced group (2.84s). However, neither difference was significant, $\mathrm{t}(21)=1.58, \mathrm{p}<.20, \mathrm{t}(21)=1.73, \mathrm{p}<.10$.

A problem with a measure like average duration is that it includes extremely long durations (one was almost $20 \mathrm{~s}$ ) and there is not a clear rationale for excluding outliers. Moreover, the key measures that appear to be ecologically valid are the percentages of glance durations over $2 \mathrm{~s}$ and $2.5 \mathrm{~s}$. In addition, the percentages of glance durations that were above $2 \mathrm{~s}, 2.5 \mathrm{~s}$, and $3 \mathrm{~s}$ distinguished between the older and younger drivers in the Chan et al. (2008) study: these percentages were $20.0 \%, 10 \%$, and $6.7 \%$, respectively, for the older, experienced drivers and $56.7 \%, 45.0 \%$ and $33.3 \%$ for the younger drivers. As

indicates, there were also clear differences in the present study between the older drivers and each younger group in the percentage of time that the groups had long glances. However, it is also clear from

that there was virtually no difference between the younger drivers who had had RAPT training and the untrained younger drivers.

Although there were substantial differences between the older group and each of the two younger untrained groups on the key measures of percentages of glances greater than 2.0,2.5, and $3.0 \mathrm{~s}$ 
(12-13\%), they were substantially less than in the Wikman et al. (1998) study which was conducted in the field (30\%). In the present study, the percentages of long glances were also quite a bit higher for all three groups. For the older drivers, the percentage of glances that were over $2.0,2.5$, and $3.0 \mathrm{sec}$ were $59.6 \%, 45.1 \%$, and $33.2 \%$, whereas they were $75.5 \%, 60.5 \%$, and $46.4 \%$ for the younger untrained drivers and $71.5 \%, 60.5 \%$, and $50.4 \%$, for the younger drivers trained with RAPT. Although these $12-13 \%$ differences between the older and younger drivers were clearly smaller than in the Wikman et al. (1998) study, the percentages for each of the two younger groups were significantly greater than for the older group, $t \mathrm{~s}>4, p \mathrm{~s}<.001$.

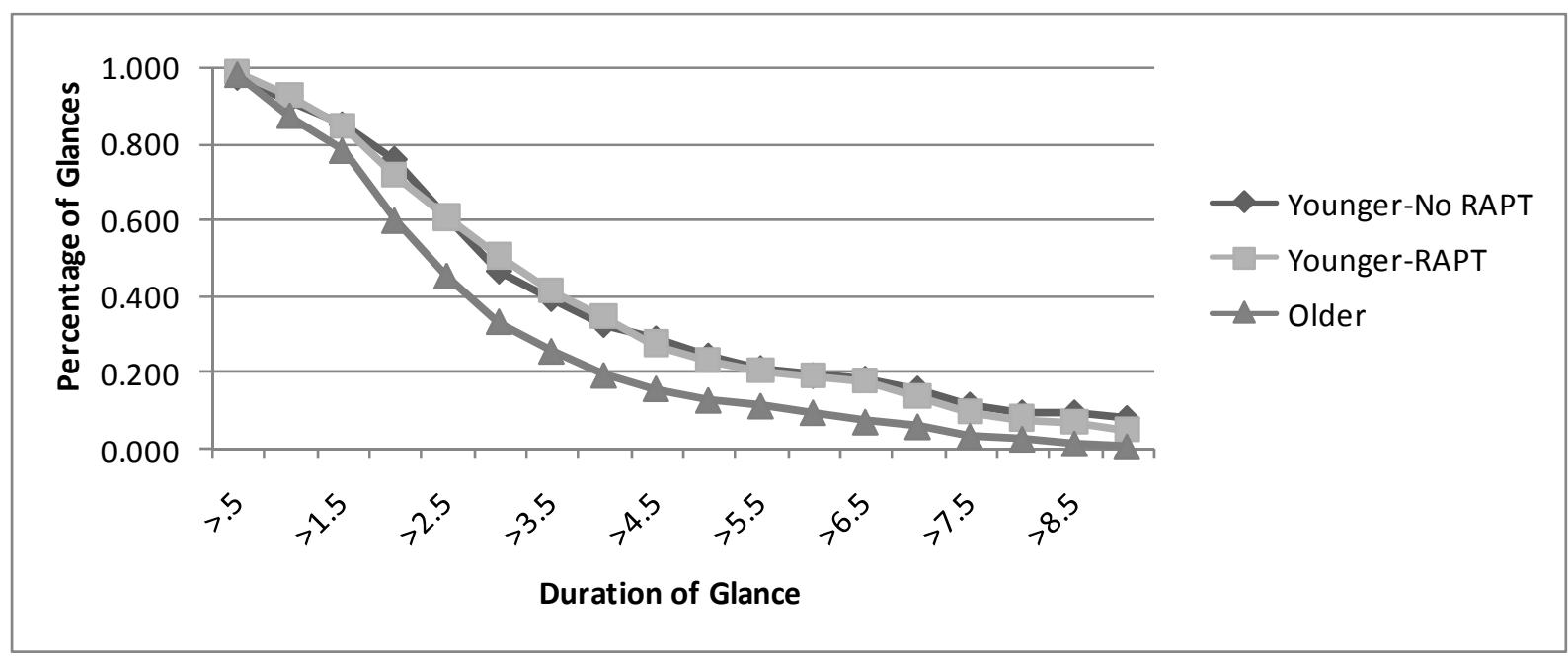

Figure 2. Percentage of Glances Greater Than Some Fixed Duration for Older, Younger RAPT and Younger no-RAPT Participants

\section{DISCUSSION}

As a start, we wanted to construct a test that had good face, construct, and predictive validity. The face validity of the task was never questioned by the participants. Indeed, most drivers will occasionally glance away from the forward roadway to look at a map. The evaluation method appeared to have good construct validity as well. There are two key indicators. First, in this study, the average glance durations at the map did not differ significantly between the younger and older drivers, just as in other studies of attention maintenance (Chan et al. 2008, Wikman et al. 1998). Second, the teen drivers in this study were more likely to glance away from the forward roadway for at least 2.0, 2.5, and 3.0 s than were older, more experienced drivers, as in other studies. However, compared to the simulator results, both the smaller differences between groups and the higher overall frequency of long glances for both older and younger drivers indicate that the environment of this test (in particular, not actually being responsible for the control of a vehicle) allows people to look away from the front roadway for longer periods of time than when they are actually driving. Finally, the evaluation method has a reasonable predictive validity, at least for the younger drivers. For our drivers using FOCAL, the percentage of glances greater than a specified duration was linearly related to the percentage of glances inside the vehicle longer than that specified duration on the field test of Wikman et al. (1998). A simple linear regression explained $99.9 \%$ of the variance $(a=-.076, b=.865)$. The percentage of glances inside the vehicle longer than some specified time on the simulator test (Chan et al., 
2008) was also a linear function of the performance on FOCAL. Thus, one can use performance on FOCAL to predict performance in the field or on a driving simulator, which ideally is what one would like from the PC based evaluation.

Given that the test has a reasonably face, construct and predictive validity, one has some reason to believe that it could be used in place of much more extensive testing on a driving simulator using an eye tracker to monitor gaze duration and location and then predict from this how well the participant would do in the simulator and on the open road. Moreover, because performance is not at ceiling for glance durations longer than $2.0 \mathrm{~s}$ and not at floor for glance durations shorter than $7.0 \mathrm{~s}$, there is a reasonable expectation that FOCAL could be used to evaluate the effects of attention maintenance training.

We had expected that the hazard anticipation program, RAPT, would produce improvements in the attention maintenance skills of the novice drivers because the experiments with RAPT (Pollatsek et al., 2006) had shown clear improvement in hazard anticipation skills. These improvements in hazard anticipation skills are arguably a consequence of the fact that novice drivers learned that it takes very active scanning in order to adequately assess threats in the environment. It would thus seem that drivers trained with RAPT should have been less willing to take their eyes away from the forward roadway. However, this did not happen (at least in the PC-environment). Towards this end, we are now developing an attention maintenance training program which we hope to evaluate on the driving simulator as well as on this instrument.

In summary, attention maintenance is increasingly a problem among all drivers as in-vehicle tasks proliferate and especially for novice drivers who are willing to glance away from the forward roadway for much longer periods of time than more experienced drivers. Driver education programs have not appeared to reduce this difference. An inexpensive program such as FOCAL that can be used to evaluate novice drivers' attention maintenance skills would give driving schools a tool to determine just how well novice drivers maintain attention.

\section{ACKNOWLEDGEMENTS}

Portions of this research were supported by grants from the National Highway Traffic Safety Administration and National Institutes of Health to Donald L. Fisher and Alexander Pollatsek.

\section{REFERENCES}

Chan, E., Pradhan, A. K., Knodler, M. A, Pollatsek, A. and Fisher, D. L. (January 2008). Empirical Evaluation on a Driving Simulator of the Effect of Distractions Inside and Outside the Vehicle on Drivers' Eye Behaviors. Proceedings of the 87th Transportation Research Board Annual Meeting CD-ROM, TRB, National Research Council, Washington, D.C.

Chisholm, S.L., Caird, J.K. and Lockhart, J. (2008). The effects of practice with MP3 players on driving performance. Accident Analysis and Prevention, 40, 704-713.

Klauer, S. G., Dingus, T. A., Neale, V. L., Sudweeks, J. D., \& Ramsey, D. J. (2006). The Impact of Driver Inattention On Near-Crash/Crash Risk: An Analysis Using the 100-Car Naturalistic Driving Study Data. Washington, D.C.: National Highway Traffic Safety Administration. 
Lee, S. E., Olsen, E. C. B., \& Simons-Morton, B. (2006). Eyeglance Behavior of Novice Teen and Experienced Adult Drivers. Transportation Research Record, 1980, 57-64.

Lerner N., and Boyd, S. (2004). Task Report: On-Road Study of Willingness to Engage in Distracting Tasks. Report Jointly Funded Under National Highway Safety Administration (Contract DTNH22-99-D-07005, Task Order Number 7) and National Institute of Child Health and Human Development (Contract GS-23F-8144H). Westat, Rockville: MD.

Pollatsek, A., Narayanaan, V., Pradhan, A., and Fisher, D. L. (2006). The Use of Eye Movements to Evaluate the Effect of PC-Based Risk Awareness Training on an Advanced Driving Simulator. Human Factors, 48, 447-464.

Pradhan, A. K., Pollatsek, A., Knodler, M. and Fisher, D. L. (2009). Can younger drivers be trained to scan for information that will reduce their risk in roadway scenarios that are hard to identify as hazardous? Ergonomics, 1-17, DOI: 10.1080/00140130802550232, iFirst article

Salvucci, D.D., Markley, D., Zuber, M. and Brumby, D. P. (2007). iPod Distraction: Effects of Portable Music-Player Use on Driver Performance. CHI, San Jose, California.

Strayer, D. L. \& Drews, F. A. \& Crouch, D. J. (2003). Fatal distraction? A comparison of the cell-phone driver and the drunk driver. In D. V. McGehee, J. D. Lee, \& M. Rizzo (Eds.) Driving Assessment 2003: International Symposium on Human Factors in Driver Assessment, Training, and Vehicle Design. Published by the Public Policy Center, University of Iowa (pp. 25-30).

Wang, J. S., Knipling, R. R., and Goodman, M.J. (1996). The role of driver inattention in crashes: New statistics from the 1995 crashworthiness data system. In 40th Annual Proceedings of the Association for the Advancement of Automotive Medicine, Vancouver, British Columbia.

Wikman, A., Nieminen, T., \& Summala, H. (1998). Driving experience and time-sharing during in-car tasks on roads of different width. Ergonomics, 41(3), 358-372. 